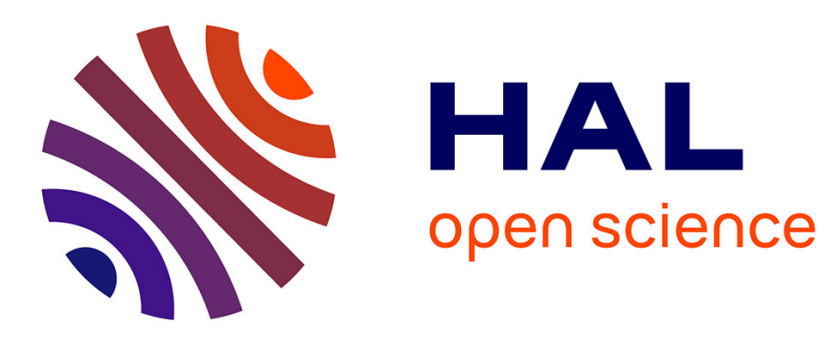

\title{
The Shapley-Shubik power index for dichotomous multi-type games
}

Sébastien Courtin, Zéphirin Nganmeni, Bertrand Tchantcho

\section{To cite this version:}

Sébastien Courtin, Zéphirin Nganmeni, Bertrand Tchantcho. The Shapley-Shubik power index for dichotomous multi-type games. Theory and Decision, 2016, 81 (3), pp.413-426. 10.1007/s11238-0169541-4 . halshs-01545769

\section{HAL Id: halshs-01545769 \\ https://shs.hal.science/halshs-01545769}

Submitted on 23 Jun 2017

HAL is a multi-disciplinary open access archive for the deposit and dissemination of scientific research documents, whether they are published or not. The documents may come from teaching and research institutions in France or abroad, or from public or private research centers.
L'archive ouverte pluridisciplinaire HAL, est destinée au dépôt et à la diffusion de documents scientifiques de niveau recherche, publiés ou non, émanant des établissements d'enseignement et de recherche français ou étrangers, des laboratoires publics ou privés. 


\title{
The Shapley-Shubik POWER INDEX FOR DICHOTOMOUS MULTI-TYPE GAMES
}

\author{
Sébastien Courtin ${ }^{\dagger}$ And Zephirin Nganmeni ${ }^{\ddagger}$ And Bertrand Tchantcho ${ }^{\S}$ \\ September 2015
}

\begin{abstract}
This work focuses on multi-type games in which there are a number of nonordered types in the input, while the output consists of a single real value. When considering the dichotomous case, we extend the Shapley-Shubik power index and provide a full characterization of this extension. Our results generalize the literature on classical cooperative games.
\end{abstract}

KEYWORDS: Game theory, Multi-type games, Simple games, Shapley-Shubik in$\operatorname{dex}$

JEL Classification Numbers: C71 ;D71.

*The authors would like to thank Fabian Gouret, Mathieu Martin, Matias Nunez and Issofa Moyouwou for their useful comments and encouragement. This work has also benefited from comments by a number of conference and seminar participants. This research has been developed within the center of excellence MME-DII (ANR-11-LBX-0023-01), and the CoCoRICo-CoDEC research program (ANR-14-CE24-0007-02).

†Université de Caen Basse-Normandie, CREM, UMR CNRS 6211, France. Email: sebastien.courtin@unicaen.fr;

‡Université de Cergy-Pontoise, THEMA, UMR CNRS 8184, France. Email: zephirin.ngameni@ucergy.fr.

§University of Yaounde I, Advanced Teachers' Training College, Cameroon; and University of Cergy-Pontoise, THEMA, UMR CNRS 8184, France. Email: btchantcho@yahoo.fr; 


\section{Introduction}

This paper is devoted to the study of multi-type games, which are a generalization of the classical cooperative game. This framework models games for which the following conditions are met: (i) Several, say $r$ non-ordered types (not levels) of support are allowed in the input. Each player chooses one of the $r$ types of support. Note that a player can also make no choice. The players' choices then lead to a choice configuration. (ii) The characteristic function maps each choice configuration to a real number, the value of the configuration.

By their nature, classical cooperative games allow two levels of inputs. For example, in a transferable utilities game, each player chooses either "to participate" or "not", and a characteristic function maps each choice configuration to a real value. In a simple voting game, when a single alternative, such as a bill or an amendment, is pitted against the status quo, the alternative is approved only on the basis of the votes cast by those who are in favor. In other words, voting "yes" and "no" are the only feasible alternatives.

It has been pointed out by many scholars that a number of interesting questions of economics, politics and more generally social sciences cannot be described by a classical cooperative game. For example, in some real voting systems such as the United Nations Security Council, the United States federal system and the Council of Ministers of the European Union, "abstention" plays a key role. However, these voting systems cannot be modeled by such games. This goes some way towards explaining the introduction of many different models of such games in recent years.

Felsenthal and Machover [1997], Felsenthal and Machover [1998] and Felsenthal and Machover [2001] introduced ternary voting games where "abstention" is permitted as a distinct third option of a voter, intermediate between a "yes" and a "no" vote. Thus, each individual voter expresses one of the three possible levels of input support, and the output consists of either collective acceptance or collective rejection. A similar model is the bicooperative game of Bilboa et al. [2000] in which the third alternative is "no participation" . Clearly, these three input supports are totally ordered in the sense that a "yes" vote is more favorable to the collective acceptance than an "abstention" vote (or a "not participating" vote), which in turn is more favorable than a "no" vote. Ternary voting games or $(3,2)$ games have been directly extended to $(j, k)$ games by Freixas and Zwicker [2003. In the $(j, k)$ simple games, each player expresses one of $j$ ordered possible levels of input support, and the out- 
put consists not of a real value but of one of $k$ possible levels of collective support. Another model of game with ordered inputs is the so-called multichoice game introduced by Hsiao and Raghavan [1993]. In these games, each player is allowed to have a given number of effort levels, each of which is assigned a nonnegative weight. The weight assigned to an effort level leads to an ordering on the set of effort levels. Any choice configuration is then associated with a real value.

An example of a model in which alternatives in the inputs are not totally ordered is the one formalized by Laruelle and Valenciano [2012]. They study quaternary voting rules in which the four possible alternatives are "yes", "no", "abstention" and "non-participation". The collective decision is dichotomous, i.e., either the proposal is accepted or rejected. Levels of support here are not totally ordered, since the "non-participation" and the "abstention" options are not ranked. Indeed, in some situations, the "abstention" option may be more favorable to the rejection of the proposal than the "non-participation" option, while in other situations the converse is observed. However, the "yes" alternative is always more favorable than any other alternative while the "no" alternative is less favorable than any alternatives. This model and the other models above are particular cases of the more general framework of games on lattices developed by Grabisch and Lange [2007]. In this model, each player $i$ has a set $L_{i}$ of possible actions and this set is endowed with a lattice, i.e., a partial order such that any pair of actions possesses a least upper bound and a greatest lower bound.

A model of a game that does not feature among the class of games on lattices is that developed by Bolger [1986], Bolger [1993], Bolger [2000] and Bolger [2002], called games with $n$ players and $r$ alternatives, or simply $r$-games. In such games, there are $r$ possible input alternatives that are not ordered. Each alternative $j$ attracts its own coalition of supporting voters. A configuration, which is a partition of the set of players into $r$ subsets (some of which might be empty), is then associated with an $r$-tuple of cardinal values. The component $j$ represents the value of the coalition of the configuration that has chosen the input $j$. This model is related to ours in the sense that the set of inputs is not ordered. No alternative is a priori more favorable than another. However, the models differ in their outputs. Our output consists of a single value. It is worth noting that our model is less general than that of Monroy and Fernandez [2009], who introduced multi-criteria simple games. Indeed, in their framework, no ordering is considered in the input or in the output, and furthermore more than one alternative can be chosen by each player. 
As usual, a central concern in game theory is to define a value or solution concept for a game. The prominent value is the well-known Shapley value. This value for multichoice cooperative games is due to Hsiao and Raghavan [1993]. More generally, Grabisch and Lange [2007] extend the Shapley value to all games on lattices. The corresponding value for $r$-games was developed by Bolger [1993], and later extended by Amer et al. [1998] and Magana [1996].

In the particular context of simple games, different theories of power have been proposed. The most famous is the Shapley-Shubik (Shapley and Shubik [1954]) voting power index. This index has been extended to the context of multiple alternatives in various games. It was defined for ternary voting games by Felsenthal and Machover [1997]. For $(j, k)$ games the extension is due to Freixas [2005].

Our main contribution is to extend and fully characterize the Shapley-Shubik index, when "dichotomous" multi-type games $(D M G)$ are considered. DMG are particular cases of multi-type games where the output is dichotomous.

This work is structured as follows. Section 2 introduces the general framework of multi-type games and some examples. Section 3 defines and characterizes the Shapley-Shubik power index for DMG. Finally, Section 4 extends our analyze to the Banzhaf power index and concludes the paper.

\section{General framework of multi-type games}

\section{Classical cooperative game}

A finite set of players is denoted by $N=\{1,2, \ldots, n\}, \wp(N)$ is the set of all subsets of $N$ and $2^{N}$ is the set of all nonempty subsets of $N: 2^{N}=\wp(N) \backslash\{\varnothing\}$. We refer to any subset $S$ of $N$ as a coalition. A classical cooperative $n$-person game in characteristic form is defined by a function $v: \wp(N) \rightarrow \mathbb{R}$, such that $v(\varnothing)=0$. In this paper we consider monotonic cooperative $n$-person games, i.e. cooperative $n$-person games satisfying $v(S) \leq v(T)$ if $S \subseteq T \subseteq N$.

We will now generalize classical cooperative games to multi-type games. 


\section{Multi-type games}

Let $R=\left\{a_{1}, \ldots, a_{k}, \ldots, a_{r}\right\}$, where $r$ is a non-null integer, be the set of types of support the players can choose ${ }^{1}$. Denote $a_{0}$ the option which means no support at all. Each player can then choose between $r+1$ possible actions : no support, or one of the $r$ types of support. Let $\bar{R}=\left\{a_{0}, a_{1}, \ldots, a_{k}, \ldots, a_{r}\right\}$ bet the set of all possible actions. A configuration is a sequence $F=\left(F_{k}\right)_{k \in \bar{R}}$, such that for all $k \in \bar{R}, F_{k} \subseteq N$ and for all $k, j \in \bar{R}, k \neq j \Longrightarrow F_{k} \cap F_{j}=\varnothing$. F can be seen as a division of the voters according to their action, while $F_{k}$ is the set of voters who choose the action $k \in \bar{R}$. We denote by $\bar{R}^{N}$ the set of all configurations and by $\varnothing^{r}$ the configuration defined by : $\varnothing^{r}=$ $(N, \varnothing, \ldots, \varnothing)$. In words, $\varnothing^{r}$ represents the situation where no player chooses one of the support.

A multi-type game is a pair $(N, V)$ where $V: \bar{R}^{N} \rightarrow \mathbb{R}$, such that $V\left(\varnothing^{r}\right)=0$.

Let $F=\left(F_{0}, F_{1}, \ldots, F_{r}\right)$ be a configuration. Then it is clear that $F_{0}=N-\bigcup_{k=1}^{k=r} F_{k}$. This means that $F$ is completely described by the given of $\left(F_{k}\right)_{1 \leq k \leq r}$. This allows us to consider in the sequel that a configuration is a sequence $\left(F_{k}\right)_{1 \leq k \leq r}$ and we will let $N_{F}=\bigcup_{k=1}^{k=r} F_{k}=\cup_{k \in R} F_{k} \cdot N_{F}$ will be referred to as the support of the configuration $F$, which is the set of voters who chooses one of the support.

Clearly a multi-type game reduces to a classical cooperative game when there is only two possible actions, one type of support i.e. when $|R|=1$.

\section{Examples}

The first example is inspired by Example 2, page 320 in Bolger [1993.

Example 1. Consider a society of $n$ individuals. This society can be divided into three categories. The first one consists of those who are working. The second one concerns those who are currently unemployed. And finally, in Category 3 we find the individuals who leave the unemployment system. If a player $i$ is employed, he can earn (annually) $w_{i}$ euros. If player $i$ is in Category 2, then he receives, as an unemployment benefit, 70 percent of his potential earning, namely $0.7 w_{i}$. Finally individuals in Category 3 receive a minimal income, i.e., 30 percent of their potential income.

A configuration $F$ can be seen as a couple $F=\left(F_{1}, F_{2}\right)$ where: $F_{1}$ is the set of individuals in category $1 ; F_{2}$ is the set of individuals in category 2 ; and $N \backslash\left(F_{1} \cup F_{2}\right)$ is the set of

\footnotetext{
${ }^{1}$ For the sake of simplicity and when there is no ambiguity, we write $k \in R$ for an element $a_{k} \in R$.
} 
individuals in category 3. The value of a given configuration can be thought of as the "wage income" of the society, that is the sum of incomes of each category.

The second example deals with non-hierarchical multitask organizations, inspired by Pongou et al. [2015].

Example 2. Consider an organization in which there is a nonempty set of workers and a collection of finite sets of tasks. A real-valued production function maps each allocation of workers across the tasks to a real number which measures the aggregate output or productivity of the organization under that particular configuration.

Note that the configuration of workers may lead to a product of good or bad quality. In these special situations the output is dichotomous, as in the following example.

The third example concerns the particular case of dichotomous multi-type games and is adapted from Freixas [2005] and Hsiao and Raghavan [1993].

Example 3. Consider a committee that must decide on the promotion of a certain junior colleague in an economics department. There are three full professors (namely 1, 4 and 5) and two associate professors (namely 2,3) on this committee. Each of the professors may express a Research Support (RS), a Teaching Support (TS) or an Administrative Support (AS). The candidate will be selected only when both of the following conditions are met:

1. At least two full professors express a Research Support.

2. One or more associate professors expresses at least one of the supports.

The multi-type game associated with this situation is such that $R=\{R S, T S, A S\}$.

Two final remarks. First, a multi-type game $(N, V)$ is said to be monotonic if for all $F, G \in R^{N}, F \subseteq G \Longrightarrow V(F) \leq V(G)$ 2. Strictly speaking, a multi-type game need not be monotone. Indeed, in some contest, monotonicity may seem not natural. However, for the purpose of simplicity, the multi-type games considered in this paper are monotonic. Second, there is not restriction about the independence of the supports and these support levels are not categorized by importance.

We will now focus on dichotomous games. In the classical framework, dichotomous game are represented by simple games.

\footnotetext{
${ }^{2}$ Note that $F \subseteq G$ if for all $k \in R, F_{k} \subseteq G_{k}$.
} 


\section{Simple voting games}

A simple (voting) game (SG) consists of a pair $(N, v)$ such that: (i) $v(S) \in\{0,1\}$ for all $S \in \wp(N)$; (ii) $v(\emptyset)=0$; (iii) $v(N)=1$; and (iv) $v$ is monotonic. A coalition $S$ is said to be winning in $v$ if $v(S)=1$, and losing otherwise. An $S G$ is said to be anonymous if there exists a non-null integer $q$ such that for all $S \in 2^{N}: v(S)=1$ iff $|S| \geq q$. In an anonymous $S G$ the outcome depends solely on the number of the "yes" votes rather than on their individual identities.

In the following, for simplicity an $S G(N, v)$ will be denoted by $v, S \cup\{i\}$ will be written $S+i$, while $S \backslash\{i\}$ represents $S-i$.

\section{Dichotomous multi-type games}

As a natural extension of $S G$, a dichotomous multi-type $(D M G)$ is given by a map $V: R^{N} \rightarrow \mathbb{R}$ such that: (i) $V(F) \in\{0,1\}$ for all $F \in R^{N}$; (ii) $V(F)=1$ for at least one $F \in R^{N}$; and (iii) $V$ is monotonic. A configuration $F$ is a winning configuration for $V(F)=1$ while it is a losing configuration for $V(F)=0$.

A DMG is anonymous if there exists a vector of integers $\left(q_{k}\right)_{k \in R}$ such that for all $F \in R^{N}: V(F)=1 \Leftrightarrow$ for all $k \in R,\left|F_{k}\right| \geq q_{k}$. This means that the number of voters who choose each support $k$ is sufficient to determine the outcome.

Once again, the dichotomous multi-type game reduces to a simple game when there is only one type of support.

The main problem is to define a value for multi-type games. However, in this paper we restrict ourselves to $D M G$. In this context, a value refers to a power index.

\section{Power index: $S G$}

A power index is a mapping $\varphi$ assigning to each $S G$ an $n$-dimensional real-valued vector $\varphi(v)=\left(\varphi_{1}(v), \ldots, \varphi_{n}(v)\right)$. $\varphi_{i}(v)$ can be interpreted as the voting power of player $i \in N$ in game $v$.

In general, the classical power indices can be summarized by the following for- 
mula: 3

$$
\varphi_{i}(v)=\sum_{S \in 2^{N}, i \in S} \alpha(i, S)[v(S)-v(S-i)]
$$

The marginal contribution of a player $i$ belonging to a coalition $S$ is given by $v(S)-v(S-i)$. If $v(S)-v(S-i)=1$, player $i$ is said to be decisive in $S$.

$\alpha(i, S)$ can be seen as a weight which depends on the coalition $S \in 2^{N}$ and on the player $i$. However, in most of these indices, $\alpha(i, S)$ does not depend on $i$ but solely on $S$. This is the case, for example, with:

- Johnston (Johnston $[1978])$, for which $\alpha(i, S)=\frac{1}{d(S)}$ with $d(S)$ being the number of decisive players in $S$;

- Shapley-Shubik (Shapley and Shubik [1954]), for which $\alpha(i, S)=\frac{(|S|-1) !(n-|S|) !}{n !}$;

- Banzhaf (Banzhaf $[1965])$, for which $\alpha(i, S)=\frac{1}{2^{n-1}}$.

We call this class of power indices weight-dependent power indices. In what follows, a weight-dependent power index for a player $i$ in a $S G v$ is then given by:

$$
\varphi_{i}(v)=\sum_{S \in 2^{N}} \alpha_{S}[v(S)-v(S-i)],
$$

with $\alpha_{S}$ the weight of coalition $S$.

\section{Power index in $D M G$}

We first propose a natural extension of a decisive player to $D M G$.

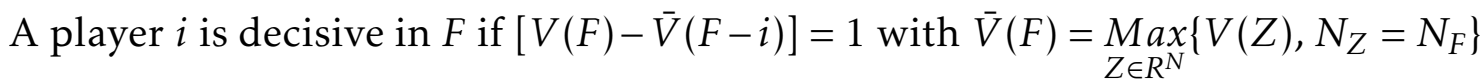
and $F-i=\left(F_{k}-i\right)_{k \in R}$. In words, a player $i$ is decisive in a configuration $F$ if $F$ is a winning configuration and whatever the types of support of the other voters, when he decides to not support the proposal, the winning configuration becomes a losing one.

From this definition, we derive a generalization of weight-dependent power indices, in the context of DMG. A weight-dependent power index for a player $i$ in a

\footnotetext{
${ }^{3}$ There is a large literature on the many notions of power indices (see Andjiga et al. [2003] and Laruelle and Valenciano [2008] for a detailed description of these different notions).
} 
$D M G$ is in the form :

$$
\Psi_{i}(V)=\sum_{F \in R^{N}} \alpha_{F}[V(F)-\bar{V}(F-i)]
$$

where $\alpha_{F}$ is referred to as the weight of the configuration $F$.

Note that for $|R|=1$, a weight-dependent power index for a $D M G$ is equivalent to a weight-dependent power index for an SG.

In the following, we focus on the most important power index, i.e. the ShapleyShubik power index.

\section{Shapley-Shubik power index for $D M G$}

In this section, we outline an axiomatic approach for the Shapley-Shubik power index for $D M G$. There is a large literature on the characterization of this index for $S G$. Below, we provide a characterization of the Shapley-Shubik power index in the class of weight-dependent power indices for DMG. The first axiom is a sort of amalgamation of the classical efficiency and symmetry conditions.

Axiom 1. Equal Share for Anonymous Game (ESAG):

$\Psi$ satisfies ESAG if for all anonymous DMG $V$ and all $i \in N, \Psi_{i}(V)=\frac{1}{n}$.

We introduce below another property for weight-dependent power indices.

Axiom 2. Equal Size (ES) :

$\Psi$ satisfies ES if for all $F, G \in R^{N}$, and all $k \in R,\left|F_{k}\right|=\left|G_{k}\right|$ implies $\alpha_{F}=\alpha_{G}$.

In any anonymous $D M G$, any player has exactly the same power which is given by $\frac{1}{n}$. According to the ES condition, all configurations must be equally weighted, provided that only the number of players in a given type of support matters.

The following theorem generalizes the Shapley-Shubik power index to DMG.

Theorem 1. There exists exactly one weight-dependent power index satisfying the ESAG and $\mathrm{ES}$ axioms for DMG, the weight of which for a configuration $F$ is given by:

$$
\alpha_{F}=\frac{\left(n-\sum_{k \in R}\left|F_{k}\right|\right) !\left(\prod_{k \in R}\left|F_{k}\right| !\right)}{n !\left(\sum_{k \in R}\left|F_{k}\right|\right)}
$$


In order to prove Theorem 1 above, the following results will be useful.

The first result is straightforward from combinatorial analysis

Lemma 1. Let $N$ be a set of $n$ players and $\left(n_{t}\right)$ a sequence of $r$ integers such that $\sum_{t=1}^{r} n_{t}=n$. The number of partitions of $N$ into $r$ coalitions $S_{1}, \ldots, S_{r}$ such that for all $t=1,2, \ldots, r$ $\left|S_{t}\right|=n_{t}$ is given by the formula $\frac{n !}{n_{1} ! \times n_{2} ! \times \ldots \times n_{r} !}$.

The next result characterizes a decisive player in an anonymous DMG.

Lemma 2. Let $V$ be an anonymous DMG in which for all $F \in R^{N}, V(F)=1$ iff for all $k \in R,\left|F_{k}\right| \geq q_{k}$. Let $F \in R^{N}:$ a voter $i \in N$ is decisive in $F$ if and only if $i \in N_{F}$ and for all $k \in R,\left|F_{k}\right|=q_{k}$.

Proof. Let $V$ be an anonymous $D M G$ in which for all $F \in R^{N}, V(F)=1 \Leftrightarrow$ for all $k \in R,\left|F_{k}\right| \geq q_{k}$.

Let $F \in R^{N}$ and a voter $i \in N$.

$\Rightarrow$ ) Assume that $i$ is decisive in $F$. Then by definition, $i \in N_{F}$.

Assume now that there exists $j \in R$ such that $\left|F_{j}\right|>q_{j}$.

- If $i \in F_{j}$ then $\left|F_{j}-i\right|=\left|F_{j}\right|-1 \geq q_{j}$; furthermore, $\forall k \in R-j,\left|F_{k}-i\right|=\left|F_{k}\right| \geq q_{k}$. It follows that $V(F-i)=1$ and consequently $\bar{V}(F-i)=1$. This is a contradiction since $i$ is decisive in $F$.

- If $i \notin F_{j}$; consider a given player $i^{\prime} \in F_{j}$, set $i \in F_{p}$ with $p \neq j$ and consider the configuration $G$ defined as follows :

for all $k \in R, G_{k}=\left\{\begin{array}{l}F_{k} \text { if } k \notin\{p, j\} \\ F_{k}-i^{\prime} \text { if } k=j \\ \left(F_{k}-i\right)+i^{\prime} \text { if } k=p\end{array}\right.$

It is straightforward that $\forall k \in R,\left|G_{k}\right| \geq q_{k}$, that is, $V(G)=1$ and $\bar{V}(F-i)=1$ since $N_{G}=N_{F}-i$. Once again, this is a contradiction since $i$ is decisive in $F$. It then follows that $\forall k \in R,\left|F_{k}\right|=q_{k}$.

$\Leftarrow)$ Let $F \in R^{N}$ with $i \in N_{F}$ and $\forall k \in R,\left|F_{k}\right|=q_{k}$.

Since $\left|F_{k}\right|=q_{k}, V(F)=1$ and $\left|N_{F}\right|=\left|\bigcup_{k \in R} F_{k}\right|=\sum_{k \in R} q_{k}$. Let $G \in R^{N}$ such that $N_{G}=$ $N_{F}-i$; then $\left|N_{G}\right|=\left|N_{F}\right|-1$ and $\left|N_{G}\right|<\left|N_{F}\right|$. Thus, $\sum_{k \in R}\left|G_{k}\right|-\sum_{k \in R} q_{k}<0$, which implies that $\sum_{k \in R}\left(\left|G_{k}\right|-q_{k}\right)<0$, and therefore there exists a $j \in R$ such that $\left|G_{j}\right|<q_{j}$. This 
leads to $V(G)=0$ and $\bar{V}(F-i)=0$. Finally, $i$ is decisive in $F$, because $V(F)=1$ and $\bar{V}(F-i)=0$.

We can now prove Theorem 1 .

Proof. $\Rightarrow$ ) Let $\Psi$ be a weight-dependent power index for $D M G$ associated with the weights $\left(\alpha_{F}\right)$ satisfying the ESAG and ES axioms. Let $F \in R^{N}$.

We shall prove that $\alpha_{F}=\frac{\left(n-\sum_{k \in R}\left|F_{k}\right|\right) !\left(\prod_{k \in R}\left|F_{k}\right| !\right)}{n !\left(\sum_{k \in R}\left|F_{k}\right|\right)}$.

Define the DMG $V$ as follows: $\forall G \in R^{N}, V(G)=1 \Leftrightarrow \forall k \in R,\left|G_{k}\right| \geq\left|F_{k}\right|$. Clearly, $V$ is an anonymous $D M G$.

We have $\Psi_{i}(V)=\sum_{G \in R^{N}} \alpha_{G}[V(G)-\bar{V}(G-i)]=\sum_{G \in W_{R}^{i}} \alpha_{G}$

where $W_{R}^{i}=\left\{G \in R^{N}: V(G)-\bar{V}(G-i)=1\right\}$. By Lemma 2, if $i$ is decisive in $G$ then $i \in N_{G}$ and $\forall k \in R,\left|G_{k}\right|=\left|F_{k}\right|$, that is,

$V(G)-\bar{V}(G-i)=1 \Longrightarrow \alpha_{G}=\alpha_{F}$, thanks to the ES axiom.

Therefore, $\Psi_{i}(V)=\sum_{G \in W_{R}^{i}} \alpha_{F}=\alpha_{F}\left|W_{R}^{i}\right|$.

We have

$$
\begin{aligned}
W_{R}^{i} & =\left\{G \in R^{N}: V(G)-\bar{V}(G-i)=1\right\} \\
& =\left\{G \in R^{N}: i \in N_{G} \text { and } \forall k \in R,\left|G_{k}\right|=\left|F_{k}\right|\right\} \\
& =\left\{G \in R^{N}: i \in\left(\bigcup_{j \in R} G_{j}\right) \text { and } \forall k \in R,\left|G_{k}\right|=\left|F_{k}\right|\right\} \\
& =\bigcup_{j \in R}\left\{G \in R^{N}: i \in G_{j} \text { and } \forall k \in R,\left|G_{k}\right|=\left|F_{k}\right|\right\} \\
& =\bigcup_{j \in R} W_{R}^{i, j}, \text { where } W_{R}^{i, j}=\left\{G \in R^{N}: i \in G_{j} \text { and } \forall k \in R,\left|G_{k}\right|=\left|F_{k}\right|\right\}
\end{aligned}
$$

Thus, $\Psi_{i}(V)=\alpha_{F}\left|W_{R}^{i}\right|=\alpha_{F}\left|\cup_{j \in R} W_{R}^{i, j}\right|=\alpha_{F} \sum_{j \in R}\left|W_{R}^{i, j}\right|$ since

$\forall k, j \in R, k \neq j \Longrightarrow W_{R}^{i, k} \cap W_{R}^{i, j}=\varnothing$. We have $\Psi_{i}(V)=\alpha_{F} \sum_{j \in R}\left|W_{R}^{i, j}\right|$.

$\left|W_{R}^{i, j}\right|$ is exactly the number of partitions of $N-i$ into $r+1$ coalitions $G_{1}, G_{2}, \ldots$, $G_{r}$ and $G_{r+1}$ such that $\left|G_{1}\right|=\left|F_{1}\right|, \ldots,\left|G_{j-1}\right|=\left|F_{j-1}\right|,\left|G_{j}\right|=\left|F_{j}\right|-1,\left|G_{j+1}\right|=\left|F_{j+1}\right|, \ldots$, $\left|G_{r}\right|=\left|F_{r}\right|$ and $\left|G_{r+1}\right|=\left(n-\sum_{k \in R}\left|F_{k}\right|\right)$. Thanks to Lemma 1 we obtain : 


$$
\begin{aligned}
\left|W_{R}^{i, j}\right| & =\frac{(n-1) !}{\left(n-\sum_{k \in R}\left|F_{k}\right|\right) !\left(\prod_{k \in R, k \neq j}\left|F_{k}\right| !\right)\left(\left|F_{j}\right|-1\right) !} \\
& =\frac{\left|F_{j}\right| \times(n-1) !}{\left(n-\sum_{k \in R}\left|F_{k}\right|\right) !\left(\prod_{k \in R}\left|F_{k}\right| !\right)}
\end{aligned}
$$

and hence,

$$
\begin{aligned}
\Psi_{i}(V) & =\alpha_{F} \sum_{j \in R}\left|W_{R}^{i, j}\right| \\
& =\alpha_{F} \sum_{j \in R}\left[\frac{\left|F_{j}\right| \times(n-1) !}{\left(n-\sum_{k \in R}\left|F_{k}\right|\right) !\left(\prod_{k \in R}\left|F_{k}\right| !\right)}\right] \\
& =\alpha_{F} \frac{(n-1) !\left(\sum_{j \in R}\left|F_{j}\right|\right)}{\left(n-\sum_{k \in R}\left|F_{k}\right|\right) !\left(\prod_{k \in R}\left|F_{k}\right| !\right)}
\end{aligned}
$$

Thanks to the ESAG of the index $\Psi$, we have $\Psi_{i}(V)=\frac{1}{n}$ since $V$ is anonymous.

Finally, $\alpha_{F} \frac{(n-1) !\left(\sum_{j \in R}\left|F_{j}\right|\right)}{\left(n-\sum_{k \in R}\left|F_{k}\right|\right) !\left(\prod_{k \in R}\left|F_{k}\right| !\right)}=\frac{1}{n}$ which yields $\alpha_{F}=\frac{\left(n-\sum_{k \in R}\left|F_{k}\right|\right) !\left(\prod_{k \in R}\left|F_{k}\right| !\right)}{n(n-1) !\left(\sum_{j \in R}\left|F_{j}\right|\right)}=\frac{\left(n-\sum_{k \in R}\left|F_{k}\right|\right):\left(\prod_{k \in R}\left|F_{k}\right| !\right)}{n !\left(\sum_{j \in R}\left|F_{j}\right|\right)}$.

This concludes the first part of the proof.

$\Leftarrow)$ Conversely, let $\Psi$ be a power index such that $\forall F \in R^{N}, \alpha_{F}=\frac{\left(n-\sum_{k \in R}\left|F_{k}\right|\right) !\left(\prod_{k \in R}\left|F_{k}\right| !\right)}{n !\left(\sum_{k \in R}\left|F_{k}\right|\right)}$.

a) It is obvious that $\Psi$ satisfies the ES condition.

b) Let us now prove that $\Psi$ satisfies ESAG.

Consider an anonymous $D M G$ for which $\forall F \in R^{N}, V(F)=1 \Leftrightarrow \forall k \in R,\left|F_{k}\right| \geq q_{k}$.

We have $\Psi_{i}(V)=\sum_{F \in R^{N}} \alpha_{F}(V(F)-\bar{V}(F-i))=\sum_{F \in W_{R}^{i}} \alpha_{F}$

where $W_{R}^{i}=\left\{F \in R^{N}: V(F)-\bar{V}(F-i)=1\right\}$.

We know from Lemma 2 that for all $F \in W_{R}^{i}, i$ is decisive in $F$ and therefore $i \in N_{F}$ 
and $\forall k \in R,\left|F_{k}\right|=q_{k}$, which implies that $\alpha_{F}$ does not depend on $F$ and is given by $\alpha_{F}=\alpha=\frac{\left(n-\sum_{k \in R} q_{k}\right) !\left(\prod_{k \in R} q_{k}\right) !}{n !\left(\sum_{k \in R} q_{k}\right)}$; thus, $\Psi_{i}(V)=\alpha\left|W_{R}^{i}\right|$.

As we did in the first part of the proof, let us remark that:

$W_{R}^{i}=\cup_{j \in R} X_{R}^{i, j}$, where $X_{R}^{i, j}=\left\{F \in W_{R}^{i}: i \in F_{j}\right\}$.

$\left|X_{R}^{i, j}\right|$ is the number of partitions of $N-i$ into $r+1$ coalitions $F_{1}, F_{2}, \ldots, F_{r}$ and $F_{r+1}$ such that $\left|F_{1}\right|=q_{1}, \ldots,\left|F_{j-1}\right|=q_{j-1},\left|F_{j}\right|=q_{j}-1$, (by assuming that $\left.i \in F_{j}\right),\left|F_{j+1}\right|=q_{j+1}$, ..., $\left|F_{r}\right|=q_{r}$ and $\left|F_{r+1}\right|=\left(n-\sum_{k \in R}\left|F_{k}\right|\right)$.

Thanks to Lemma 1 we obtain:

$\left|X_{R}^{i, j}\right|=\frac{(n-1) !}{\left(n-\sum_{k \in R} q_{k}\right) !\left(\prod_{k \in R, k \neq j} q_{k} !\right)\left(q_{j}-1\right) !}$ and hence $\left|W_{R}^{i}\right|=\sum_{j \in R} \frac{q_{j}(n-1) !}{\left(n-\sum_{k \in R} q_{k}\right) !\left(\prod_{k \in R} q_{k} !\right)}=\frac{(n-1) !}{\left(n-\sum_{k \in R} q_{k}\right) !\left(\prod_{k \in R} q_{k} !\right)} \sum_{j \in R} q_{j}$.

Finally

$$
\begin{aligned}
\Psi_{i}(V) & =\alpha \times \frac{(n-1) !\left(\sum_{j \in R} q_{j}\right)}{\left(n-\sum_{k \in R} q_{k}\right) !\left(\prod_{k \in R} q_{k} !\right)} \\
& =\frac{\left(n-\sum_{k \in R} q_{k}\right) !\left(\prod_{k \in R} q_{k} !\right)}{n !\left(\sum_{k \in R} q_{k}\right)} \times \frac{(n-1) !\left(\sum_{j \in R} q_{j}\right)}{\left(n-\sum_{k \in R} q_{k}\right) !\left(\prod_{k \in R} q_{k} !\right)} \\
& =\frac{1}{n} .
\end{aligned}
$$

We conclude that $\forall i \in N, \Psi_{i}(V)=\frac{1}{n}$, and $\Psi$ satisfies $E S A G$.

The proof is now complete.

The Shapley-Shubik index of a player in a $D M G V$ is then defined by:

$$
S S_{i}{ }^{r}(V)=\sum_{F \in R^{N}} \frac{\left(n-\sum_{k \in R}\left|F_{k}\right|\right) !\left(\prod_{k \in R}\left|F_{k}\right| !\right)}{n !\left(\sum_{k \in R}\left|F_{k}\right|\right)}[V(F)-\bar{V}(F-i)]
$$

Let us mention that when $|R|=1$ our formula clearly reduces to that of ShapleyShubik for SG. Therefore we can deduce from Theorem 1 an alternative characteri- 
zation of Shapley-Shubik for SG.

Corollary 1. There exists one and only one weight-dependent power index for SG satisfying the ESAG and ES axioms, which is the Shapley-Shubik power index.

Note that we can also provide a characterization of the class of weight-dependent power indices based on the famous Banzhaf score (Banzhaf [1965]). Given an DMG $V$, the Banzhaf score of a player $i$ in $V$, denoted by $\eta_{i}(V)$, is the number of coalitions in which he is decisive in an DMG. More formally, for all $i \in N, \eta_{i}^{r}(V)=$ $\sum_{F \in R^{N}}[V(F)-\bar{V}(F-i)]$.

In order to provide a characterization of power indices based on the Banzhaf score, let us introduce the following axiom, referred to as the Constant-Weight axiom

Axiom 3. Constant-Weight axiom (CW):

A weight-dependent power index satisfies the $\mathrm{CW}$ axiom for DMG if there exists a constant $\delta>0$ such that for all $F \in R^{N}, \alpha_{F}=\delta$.

If this is the case then the power index is said to be a $\delta$-constant-weight power index.

We obtain the following proposition.

Proposition 1. A weight-dependent power index $\Psi$ is $\delta$-constant-weight if and only if for all DMG $V$, for all $i \in N, \Psi_{i}(V)=\delta \eta_{i}^{r}(V)$.

Proof. Let $\Psi$ be a weight-dependent power index for a DMG. If $\Psi$ is $\delta$-constantweight, then for all $i \in N$,

$$
\begin{aligned}
\Psi_{i}(V) & =\sum_{F \in R^{N}} \alpha_{S}[V(F)-\bar{V}(F-i)] \\
& =\sum_{F \in R^{N}} \delta[V(F)-\bar{V}(F-i)] \\
& =\delta \sum_{F \in R^{N}}[V(F)-\bar{V}(F-i)] \\
& =\delta \eta_{i}^{r}(V) .
\end{aligned}
$$

$\Leftarrow)$ The converse is straighforward.

Note that, for $\delta=\frac{1}{(r+1)^{n-1}}$, we obtain a generalization of Banzhaf power index introduce by Banzhaf [1965], i.e.

$$
B z_{i}^{r}(V)=\frac{\sum_{F \in R^{N}}[V(F)-\bar{V}(F-i)]}{(r+1)^{n-1}}
$$


To conclude, let us evaluate the Shapley-Shubik and the Banzhaf power index for the $D M G$ defined in Example 3 dealing with the promotion of a junior professor.

Example 4. (example 3 continued)

i) In an SG context, the professors only have to say if they are "for" or "against" the promotion. The candidate will be selected when at least two full professors and at least one associate professor vote "yes". Therefore with Shapley Shubik, $S S(v)=\left(\frac{7}{30}, \frac{9}{60}, \frac{9}{60}, \frac{7}{30}, \frac{7}{30}\right)$; while with the non-normalized Banzhaf, $B z(v)=\left(\frac{3}{8}, \frac{1}{4}, \frac{1}{4}, \frac{3}{8}, \frac{3}{8}\right)$.

ii) Consider two configurations in the DMG context: $F^{1}=(\{1,5\},\{23\},\{4\})$ and $F^{2}=$ $(\{1\},\{23\},\{\varnothing\})$. In $F^{1}, 1$ and 5 support RS, 2 and 3 choose TS and 4 chooses AS; whereas in $F^{2}$ the individuals 4 and 5 make no choice and 1, 2 and 3 continue to select the same support. Therefore $V\left(F^{1}\right)=1$ and $V\left(F^{2}\right)=0$.

Is individual 1 decisive in $F^{1}$ ? The answer is No. Indeed if we consider the configuration $G=(\{4,5\},\{23\},\{\varnothing\})$, it is easy to show that $\bar{V}(G)=1$. Therefore 1 is not decisive in $F^{1}$. In $G$, player 4 changes his support from AS to RS, and this leads to $G$, which is a winning configuration.

Therefore in DMG, Shapley Shubik, $S S^{r}(V)=\left(\frac{5}{9}, \frac{3}{9}, \frac{3}{9}, \frac{5}{9}, \frac{5}{9}\right)$; while the non-normalized Banzhaf, $B z^{r}(V)=\left(\frac{17}{128}, \frac{12}{128}, \frac{12}{128}, \frac{17}{128}, \frac{17}{128}\right)$.

\section{Conclusion}

This paper was devoted to games in which $r$ non-ordered levels of input supports are available to players. In the particular case of a dichotomous game, the output consists of 0 or 1 . Thus any choice configuration is either winning or losing. Thanks to an alternative characterizations of the Shapley-Shubik power index in the classical context, we provide full characterizations of this famous index in our framework.

There are several ways in which (dichotomous) multi-type games could be explored in further research. We can study, first, the generalization of the Shapley value to multi-type games. Secondly, we can extend two closely related solution concepts for these games, the stable sets and the core. Another interesting direction is the extension to multi-type games of the different a priori relationships between players in the spirit of Owen [1977] and Owen [1981]. Following the works of Tchantcho et al. [2008], we can also derive the desirability relation to qualitatively compare the a priori influence of voters in a dichotomous multi-type game. 


\section{References}

R. Amer, F. Carreras, and A. Magana. Extension of values to games with multiple alternatives. Annals of Operation Research, 84:63-78, 1998. 4

N. Andjiga, F. Chantreuil, and D. Lepelley. La mesure du pouvoir de vote. Mathematiques et sciences humaines, 163:111-145, 2003. 8

J.F. Banzhaf. Weighted voting doesnt work: A mathematical analysis. Rutgers Law Review, 19:317-343, 1965. 8, 14

J.M. Bilboa, J.R. Fernandez, A. Jimenez Losada, and E. Lebron. Bicooperative games. In J.M. Bilboa, editor, Cooperative games on combinatorial structures. Kluwer, Dordrecht, 2000. 2

E.M. Bolger. Power indices for multicandidate voting games. International Journal of Game Theory, 15:175-186, 1986. 3

E.M. Bolger. A value for games with $\mathrm{n}$ players and $\mathrm{r}$ alternatives. International Journal of Game Theory, 22:319-334, 1993. 3, 4,5

E.M. Bolger. A consistent value for games with $\mathrm{n}$ players and $\mathrm{r}$ alternatives. International Journal of Game Theory, 29:93-99, 2000. 3

E.M. Bolger. Characterizations of two power indices for voting games with $\mathrm{r}$ alternatives. Social Choice Welfare, 19:709-721, 2002. 3

D.S. Felsenthal and M. Machover. Ternary voting games. International Journal of Game Theory, 26:335-351, 1997. 2, 4

D.S. Felsenthal and M. Machover. The Measurement of Voting Power: Theory and Practice, Problems and Paradoxes. Edward Elgar Publishing Limited, 1st edition, 1998. 2

D.S. Felsenthal and M. Machover. Models and reality: The curious case of the absent abstention. In M.J. Holler and G. Owen, editors, Power Indices and Coalition Formation. Kluwer Academic Press, Dordrecht, 2001. 2

J. Freixas. The Shapley Shubik power index for games with several levels of approval in the input and output. Decision Support Systems, 39:185-195, 2005. 4.6 
J. Freixas and W.S Zwicker. Weighted voting, abstention, and multiple levels of approval. Social choice and welfare, 21:399-431, 2003. 2

M. Grabisch and F. Lange. Games on lattices, multichoice games and the shapley value: a new approach. Mathematical Methods of Operations Research, 65:153-167, 2007. 3, 4

C.R. Hsiao and T.E.S. Raghavan. Shapley value for multichoice cooperative games i. Games and Economic Behavior, 5:240-256, 1993. 3, 4, 6

R. Johnston. On the measurement of power : Some reaction to laver. Environment and Planning, 10:907-914, 1978. 8

A. Laruelle and F. Valenciano. Voting and collective decision-making. Cambridge University Press, 1st edition, 2008. 8

A. Laruelle and F. Valenciano. Quaternary dichotomous voting rules. Social choice and welfare, 38:431-454, 2012. 3

A. Magana. Formacion de coaliciones en los juegos cooperativos y juegos con multiples alternativas. PhD Thesis, Mathematics Department of UPC, Spain, 1996. 4

L. Monroy and F.R. Fernandez. A general model for voting systems with multiple alternatives. Applied Mathematics and Computation, 215:1537-1547, 2009. 3

G. Owen. Values of games with a priori unions. In R. Hein and O. Moeschlin, editors, Essays in Mathematical Economics and Game Theory. Springer, New York, 1977. 15

G. Owen. Modification of the Banzhaf-Coleman index for games with a priori unions. In M.J. Holler, editor, Power, Voting, and Voting Power. Physica-Verlag, Wurzburg, 1981. 15

R. Pongou, B. Tchantcho, and N. Tedjegang. Influence, relative productivity and earning in discrete multi-task organisations. mimeo, University of Ottawa, 2015. 6

L. S. Shapley and M. Shubik. A model for evaluating the distribution of power in a committee system. Rutgers Law Review, 48:787-792, 1954. 4, 8 
B. Tchantcho, L. Diffo Lambo, R. Pongou, and B. Mbama Engoulou. Journal voters? power in voting games with abstention: Influence relation and ordinal equivalence of power theories. Games and Economic Behavior, 64:335-350, 2008. 15 